\title{
Analyses of the Anatomy of the Ureter near Ureterovesical Junction on the Unenhanced CT
}

\author{
Hiroshi Juri, Kazuhiro Yamamoto, Yoshifumi Narumi \\ Department of Radiology, Osaka Medical College, Osaka, Japan \\ Email: rad103@poh.osaka-med.ac.jp
}

Received 9 March 2016; accepted 30 May 2016; published 2 June 2016

Copyright (C) 2016 by authors and Scientific Research Publishing Inc.

This work is licensed under the Creative Commons Attribution International License (CC BY). http://creativecommons.org/licenses/by/4.0/

(c) (i) Open Access

\begin{abstract}
Purpose: There has been no report evaluating the anatomy of the ureter on CT images. The purpose of this study was to retrospectively evaluate the anatomy of the ureter near the ureterovesical junction on preoperative CT. Methods: Forty patients were analyzed the craniocaudal level of the ureterovesical junction, the transverse distance from the medial line of the body to the ureterovesical junction, the direction of the ureter from the ureterovesical junction, and the length of the ureter from the point of turning medially to the ureterovesical junction. Results: The ureterovesical junction was almost at the level of the upper or middle of the left femoral head. The mean transverse distance on the right and left side was 19.0 and $19.3 \mathrm{~mm}$. The direction of the ureter was almost posterior, lateral, and cranial, but had a wide range of angles. The mean length of the ureter on the right and left side was 24.0 and $23.9 \mathrm{~mm}$. Conclusions: We can evaluate the anatomy of the ureter near the ureterovesical junction on preoperative CT.
\end{abstract}

\section{Keywords}

Ureter, Ureterovesical Junction, CT, Anatomy

\section{Introduction}

The ureter is the tract between the renal pelvis and the urinary bladder. There have been several reports about the anatomy of the ureter [1]-[5]. The pelvic part of the ureter goes downwards and turns anteriorly and medially at the level of the ischial spine, then inserts itself obliquely via a tunnel into the posterior bladder wall [1]. After the ureter turns medially, the vesical branch of the pelvic plexus passes near the ureter before the ureter joins the bladder wall [2]. Although a technique for the evaluation of the urinary tract has been developed [6] [7], there 
has been no report evaluating the anatomy of the ureter on CT images.

Recently, an autonomic nerve-preserving operation has become one of the surgical procedures for rectal cancer [8] [9]. In this procedure, the vesical branch of the pelvic plexus is usually saved. In addition, nerve-sparing radical hysterectomy has been developed to prevent post-operative bladder dysfunction for cervical cancer [10] [11]. By these procedures, the vesical branch of the pelvic plexus can be preserved after hysterectomy, and bladder dysfunction can be avoided. However, we can not evaluate the vesical branch on preoperative CT. If we evaluate the direction of the ureters near the ureterovesical junction on preoperative $\mathrm{CT}$, we may image the nerve tract of the vesical branch of the pelvic plexus, and it may lead to be useful for surgeries on rectal and cervical cancer. The purpose of this study was to evaluate the anatomy of the ureter near the ureterovesical junction on preoperative CT.

\section{Materials and Methods}

\subsection{Patients}

This study was approved by the hospital institutional review board. Informed consent was obtained from all patients. We performed a retrospective analysis of CT images from 51 patients who underwent CT for the evaluation of hematuria or urothelial carcinomas between September 2012 and March 2013. Ten patients had hydronephrosis, and we could not detect the ureter near the ureterovesical junction on the right side in 1 patient. These patients were excluded, and remaining 40 patients (22 men, 18 women; mean age, 66.9 years; range, 45 - 83 years; mean body weight, $57.4 \mathrm{~kg}$; range, 46 - $82 \mathrm{~kg}$ ) were included in this study.

\subsection{Multidetector CT Technique}

We obtained unenhanced and enhanced-phase images for all patients on a 64-row detector CT scanner (Aquillion 64, Toshiba Medical Systems Corporation, Tokyo, Japan) at the following settings: rotation time, 0.5 second; detector collimation, $64 \times 0.5 \mathrm{~mm}$; helical pitch, 53; tube voltage, $120 \mathrm{kV}$; variable tube current determined by $\mathrm{x}, \mathrm{y} \mathrm{z}$-axis dose modulation, adjustable with auto exposure control. Acquisitions were from the top of the renal cortex to the pubic symphysis. Patients were instructed to hold their breath with tidal inspiration during scanning. The CT data on each phase were reconstructed with Filtered Back Projection with the slice thickness and reconstruction intervals of $2.0 \mathrm{~mm}$ for the axial and for the MPR images to obtain the long axis of the ureter near the ureterovesical junction on the unenhanced phase.

\subsection{Points of Evaluation}

We reviewed the unenhanced CT images using a workstation (ZIO-VGR1, Amin). We evaluated the location of the orifice of the ureterovesical junction, the direction of the ureter from the ureterovesical junction, and the length of the ureter. To describe the direction of the ureter, we used a 3D coordinate system and aligned the rectangular coordinate axes with the body axes (i.e., transverse $=x$-axis, anterioposterior $=y$-axis, and vertical $=$ $z$-axis). The positive $x$-, $y$-, and $z$-axes pointed, laterally, posteriorly and cranially, respectively. We compared the right side with the left side in all patients. In addition, we divided the patients by gender, and compared ureters of males and females. Moreover, we divided the patients into 3 groups by maximum area of the urinary bladder-group 1, the maximum area of $<1500 \mathrm{~mm}^{2}$; group 2, 1500 to $3000 \mathrm{~mm}^{2}$; and group 3, $>3000 \mathrm{~mm}^{2}-$ and compared ureters among the 3 groups. The maximum area of the urinary bladder was defined as the area when the urinary bladder was largest on axial images, and the values of the area were measured by manually drawn region of interest in a workstation. There has been no report about the bladder volume considering urination, thus we divided into 3 groups predicting that the number of patients in each group were similar.

Location of the orifice of the ureterovesical junction-The craniocaudal level of the orifice of the ureterovesical junction was specified relative to the left femoral head. The left femoral head was divided into three segments, superior, middle, and inferior, and we scored using 4-point scale ( 1 = above the left femoral head, and 2 = upper, 3 = middle, and $4=$ lower portions of the left femoral head, Figure 1(a)). For this qualitative analysis, two radiologists with 6 and 23 years of experience individually evaluated and then consensus. The transverse distance from the medial line of the body to the medial edge of the orifice of the ureterovesical junction was measured using axial images by one radiologist with 6 years of experience. We defined the medial line of the body as the y-axis line passing the pubic symphysis (Figure 1(b)). 


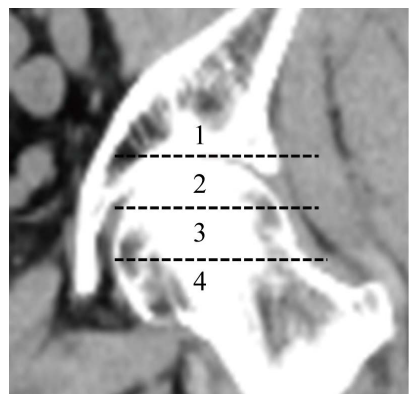

(a)

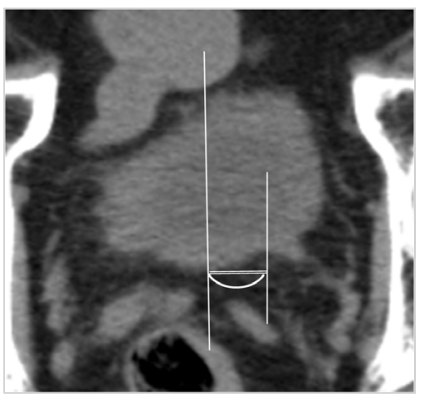

(b)

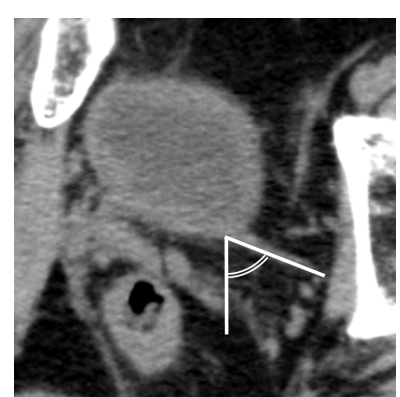

(c)

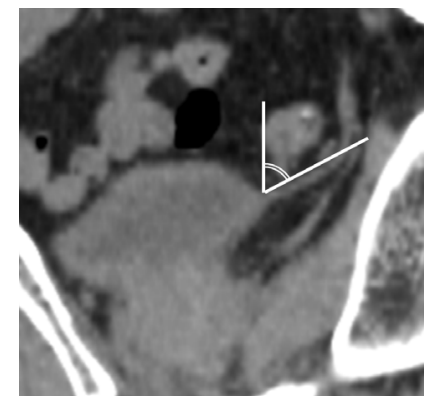

(d)

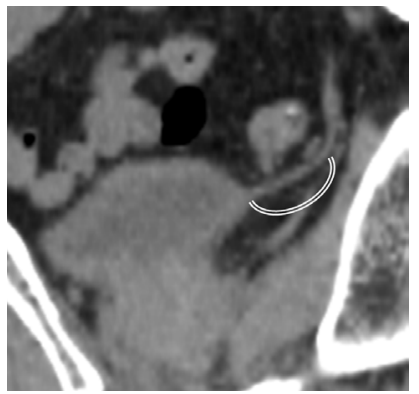

(e)

\begin{abstract}
Figure 1. Unenhanced CT was scanned and reconstructed with the slice thickness and reconstruction intervals of $2.0 \mathrm{~mm}$. (a) vertical plane shows the craniocaudal level of the orifice of the ureterovesical junction in relation to the left femoral head. The numbers indicates the score (1=above the left femoral head, and 2 = upper, 3 = middle, and 4 = lower portions of the left femoral head). (b) transverse plane seen inferiorly shows the distance from the medial line of the body to the medial edge of the orifice. (c) oblique plane seen inferiorly shows the angle $\varphi 1$. Angle $\varphi 1$ was measured positively in the posterior direction from the $x$-axis, with a negative angle being in the anterior direction. (d) vertical plane paralleling the ureter shows craniocaudal direction of the ureter from the orifice, which was measured as angle $\varphi 2$ between the ureter and the z-axis. Angle $\varphi 2$ was measured from z-axis to the ureter. (e) vertical plane paralleling the ureter shows the length of the ureter from the point of turning medially at the level of the ischial spine to the ureterovesical junction.
\end{abstract}

Direction of the ureter from the ureterovesical junction-One radiologist with 6 years of experience evaluated the points. To evaluate the direction of the ureter from the ureterovesical junction, two angles were measured: the first was the angle- -1 -between the ureter and the $y$-axis on the oblique image when the long axis of the ureter including ureterovesical junction was projected (Figure 1(c)), and the other was the angle- $\varphi 2-$ between the ureter and the z-axis when the long axis of the ureter including ureterovesical junction was projected onto the vertical plane using the MPR images (Figure 1(d)).

Length of the ureter-One radiologist with 6 years of experience evaluated the points. The length of the ureter from the point of turning medially at the level of the ischial spine to the ureterovesical junction was measured when the long axis of the ureter was projected onto the vertical plane using MPR images (Figure 1(e)).

\title{
2.4. Statistical Analysis
}

At the comparison between the right and left side, we used Wilcoxon's sign rank test for the scores of craniocaudal level of the orifice of the ureterovesical junction, and the other points of evaluation were compared by use of Student's t test. To compare males and females, we used the Mann-Whitney test for the scores of the craniocaudal level of the orifice of the ureterovesical junction, and the other points of evaluation were compared by Student's t test. For the comparison among 3 groups of the maximum bladder area, we used Kruskal-Wallis test for the scores of craniocaudal level of the orifice of the ureterovesical junction, and pairwise comparisons were performed with the Mann-Whitney test with Bonferroni correction if there were significant differences. The other points of evaluation were compared by one-way ANOVA. A value of $\mathrm{p}<0.0167$ was considered to indicate a significant difference for pairwise comparisons, and $\mathrm{p}<0.05$ was considered to indicate a significant difference 
for the others. All statistical analyses were performed with SPSS 17.0 [SPSS Inc., Chicago, IL].

\section{Results}

The number of the ureters of males and females examined were 44 and 36 . The number of patients in groups 1,2 , and 3 were 11, 18, and 11 patients, respectively. Thus, the number of ureters in group 1, 2, and 3 were 22, 36, and 22, respectively. The number of males and females in group 1, 2, and 3 were 6 and 5, 11 and 7, and 5 and 6 . The results comparing the right and left side are shown in Table 1 , the results comparing males and females are shown in Table 2, and the results comparing 3 groups of maximum bladder area are shown in Table 3.

Table 1. Points of evaluation compared between right and left sides in all patients.

\begin{tabular}{lccc}
\hline & Right & Left & p \\
\hline Location of the orifice of the ureterovesical junction & & & 0.32 \\
Craniocaudal level & $3 / 16 / 19 / 2$ & $3 / 17 / 19 / 1$ & 0.96 \\
Transverse distance (mm) & $19.0 \pm 3.9$ & $19.3 \pm 5.1$ & 0.001 \\
Direction of the ureter $\left(^{\circ}\right)$ & & $72.0 \pm 13.6$ & 0.029 \\
$\varphi 1$ & $66.4 \pm 12.6$ & $53.1 \pm 13.4$ & 0.89 \\
$\varphi 2$ & $48.6 \pm 13.0$ & $23.9 \pm 8.4$ & $24.0 \pm 9.0$ \\
Length of the Ureter $(\mathrm{mm})$
\end{tabular}

Table footnote: Data of craniocaudal level are number of patients given scores of 1, 2, 3 and 4.

Table 2. Points of evaluation compared between males and females.

\begin{tabular}{lccc}
\hline & Male & Female & $p$ \\
\hline Location of the orifice of the ureterovesical junction & & & $<0.001$ \\
Craniocaudal level & $6 / 20 / 14 / 0$ & $17.8 \pm 4.0$ & 0.054 \\
Transversedistance (mm) & $20.0 \pm 5.1$ & $63.9 \pm 7.9$ & $<0.001$ \\
Direction of the ureter $\left(^{\circ}\right)$ & & $51.8 \pm 10.8$ & 0.43 \\
$\varphi 1$ & $73.1 \pm 15.8$ & $28.5 \pm 7.8$ & $<0.001$ \\
$\varphi 2$ & $49.5 \pm 15.7$ & & \\
Length of the Ureter (mm) & $19.7 \pm 6.5$ & & \\
\hline
\end{tabular}

Table footnote: Data of craniocaudal level are number of patients given scores of 1, 2, 3 and 4.

Table 3. Points of evaluation compared among the three groups of maximum urinary bladder capacity.

\begin{tabular}{lcccc}
\hline & Group1 & Group 2 & Group 3 & p \\
\hline $\begin{array}{l}\text { Location of the orifice of the ureterovesical junction } \\
\text { Craniocaudal level }\end{array}$ & $1 / 3 / 17 / 1$ & $3 / 20 / 11 / 2$ & $2 / 10 / 10 / 0$ & 0.01 \\
Transversedistance (mm) & $16.9 \pm 3.1$ & $19.1 \pm 4.5$ & $21.5 \pm 4.6$ & 0.003 \\
Direction of the ureter $\left(^{\circ}\right)$ & & & $73.8 \pm 26.5$ & 0.071 \\
$\varphi 1$ & $64.4 \pm 6.5$ & $69.2 \pm 12.3$ & $49.2 \pm 12.4$ & 0.025 \\
$\varphi 2$ & $56.9 \pm 8.8$ & $47.3 \pm 15.2$ & $24.5 \pm 8.4$ & 0.03 \\
\hline
\end{tabular}

Table footnote: Data of craniocaudal level are number of patients given scores of 1, 2, 3 and 4 . 


\subsection{Location of the Orifice of the Ureterovesical Junction}

The score of the craniocaudal level of the orifice of the ureterovesical junction was 2 or 3 in 35 patients on the right side, and in 36 patients on the left side in all patients. The orifice was almost always located at thelevel of upper or middle portion of the left femoral head, and there was no significant difference between the right and left side ( $p=0.32)$. In contrast, the craniocaudal level of the orifice was significantly higher in males than in females $(p<0.001)$. There were significant differences among 3 groups of the maximum bladder area $(p=0.01)$. At pairwise comparisons, there were significant differences between group 1 and $2(p=0.004)$ and group 1 and $3(p=0.013)$, and there was no significant difference between group 2 and $3(p=0.69)$. The mean transverse distance from the medial line of the body to the ureterovesical junction on the right and left side was 19.0 and $19.3 \mathrm{~mm}$, and there was no significant difference $(\mathrm{p}=0.96)$. The mean transverse distance in males and females was 20.0 and $17.4 \mathrm{~mm}$, and there was no significant difference $(\mathrm{p}=0.054)$. Dividing into 3 groups of the maximum bladder area, the mean distance of groups 1, 2, and 3 was 16.9, 19.1, and $21.5 \mathrm{~mm}$, respectively, and there were significant differences $(p=0.003)$. At pairwise comparisons, there were significant differences between 1 and $3(p=0.002)$, but there was no significant difference between group between group 1 and $2(p=0.047)$, and between group 2 and $3(\mathrm{p}=0.33)$.

\subsection{Direction of the Ureter from the Ureterovesical Junction}

In all patients, the angle $\varphi 1$ ranged from $40.7^{\circ}$ to $108.4^{\circ}$ (mean, $66.4^{\circ} \pm 12.6^{\circ}$ ) on the right side, and from $50.0^{\circ}$ to $111.3^{\circ}$ (mean, $72.0^{\circ} \pm 13.6^{\circ}$ ) on the left side (Figure 2(a), Figure 2(b)). There were significant differences between the right and the left sides $(\mathrm{p}=0.001)$. The direction of the ureter from the ureterovesical junction was posterior and lateral in 38 (the right side) and 36 (the left side) patients, and was anterior and lateral in 2 (the right side) and 4 (the left side) patients. The angle $\varphi 2$ ranged from $11.6^{\circ}$ to $84.3^{\circ}$ (mean, $48.7^{\circ} \pm 13.0^{\circ}$ ) on the right side, and from $27.8^{\circ}$ to $85.4^{\circ}$ (mean, $53.2^{\circ} \pm 13.4^{\circ}$ ) on the left side (Figure 3(a), Figure 3(b)). There were significant differences between the right and left sides $(\mathrm{p}=0.029)$. The direction of the ureter was cranial in all patients. The mean angle of $\varphi 1$ in males and females was 73.1 and $63.6^{\circ}$, and there were significant differences $(p<0.001)$. The mean angle of $\varphi 2$ in males and females was 49.5 and $51.8^{\circ}$, and there was no significant difference $(p=0.43)$. Dividing into 3 groups of the maximum bladder area, the mean angles of $\varphi 1$ in group 1,2 , and 3 were $66.4,69.2$, and $71.8^{\circ}$, respectively, and there was no significant differences $(\mathrm{p}=0.071)$. The mean angle of $\varphi 2$ in group 1,2 , and 3 was $56.9,47.3$, and $49.2^{\circ}$, respectively, and there were significant differences $(\mathrm{p}=0.025)$. At pairwise comparisons, there was no significant differences between group 1 and $2(p=0.021)$ and group 2 and $3(p=0.86)$, and there were significant differences between group 1 and $3(p=0.013)$.

\subsection{Length of the Ureter}

The mean length was $24.0 \mathrm{~mm}$ on the right side, and $23.9 \mathrm{~mm}$ on the left side in all patients, and there was no

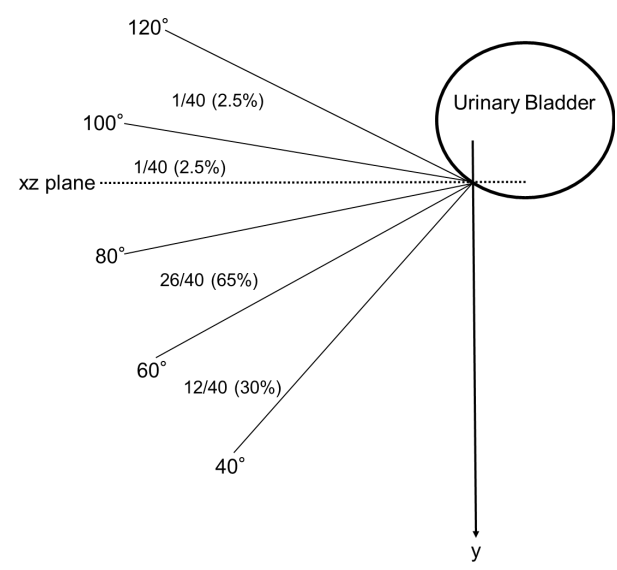

(a)

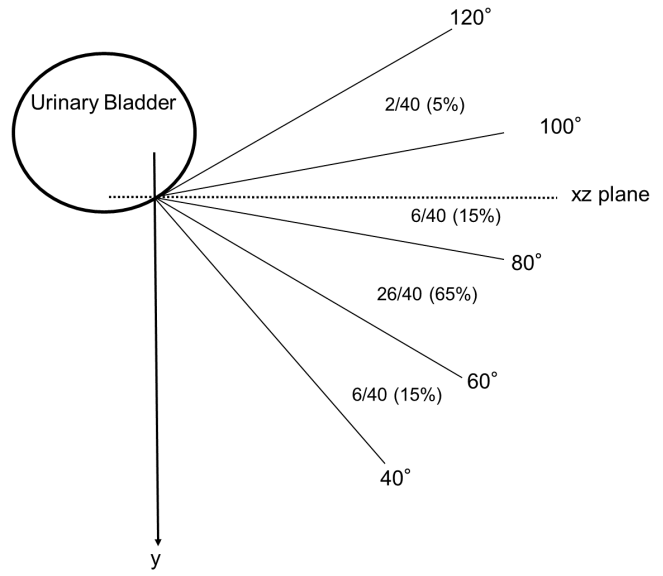

(b)

Figure 2. Direction of ureters from the orifice in the oblique plane, which is represented as the angle with the $y$-axis ( $\varphi 1$ angle). Data are numbers (\%) of ureters. (a) Right side; (b) Left side. 


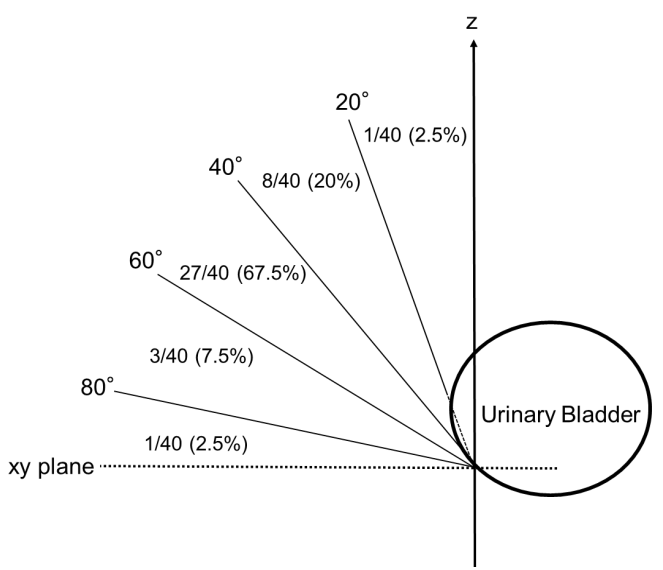

(a)

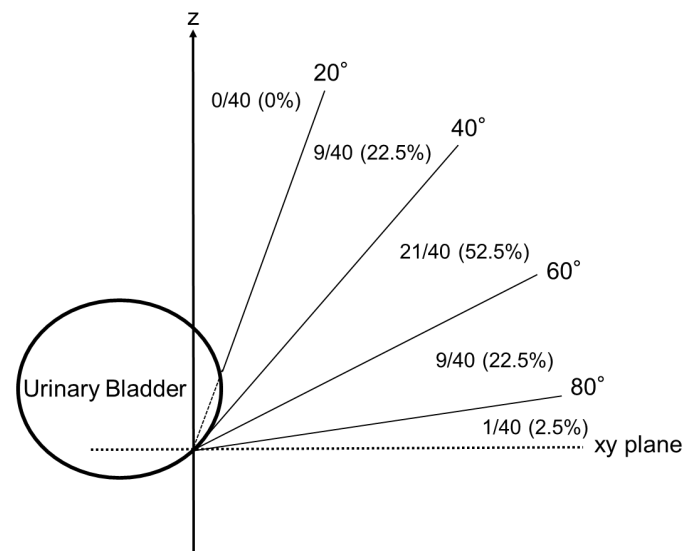

(b)

Figure 3. Direction of ureters from the orifice in the vertical plane, which is represented as the craniocaudal angle with the z-axis ( $\varphi 2$ angle). Data are numbers (\%) of ureters. (a) Right side; (b) Left side.

significant difference $(\mathrm{p}=0.89)$. The mean length in males and females was 19.7 and $28.5 \mathrm{~mm}$, and there were significant differences $(\mathrm{p}<0.001)$. Dividing into 3 groups of the maximum bladder area, the mean length in group 1, 2, and 3 was 27.0, 21.1, and $24.5 \mathrm{~mm}$, respectively, and there were significant differences $(p=0.03)$. At pairwise comparisons, there was no significant difference ( $\mathrm{p}$ values between group 1 and 2, group 1 and 3 and group 2 and 3 were $0.026,0.56$ and 0.29 ).

\section{Discussion}

Before operations for rectal or cervical cancer, surgeons almost always obtain enhanced CT. The main role of CT is to check the involvement of adjacent structures, metastases, or the vascular anatomy related to the tumor. On the other hand, in nerve-sparing surgery for patients with rectal or cervical cancer, the anatomy of the ureter is important to preserve the nerves. The vesical branch of the pelvic plexus, which is one of the nerves that should be preserved, runs near the ureter [2]. In addition, the CT is scanned in the supine position, which is the same patient position in which surgery is performed. Therefore, we think that the preoperative evaluation of the ureter on the preoperative CT may be useful for the prediction of the vesical branch of the pelvic plexus. We chose the unenhanced phase for evaluation of the ureter because there was no contrast material in the body during the operation of rectal or cervical cancer. Next, we discuss the specific points of evaluation of our study.

The craniocaudal level of the orifice of the ureterovesical junction was almost always between the level of the upper or the middle of the left femoral head on both sides. The urinary bladder is at the bottom of the pelvis; thus, this result might be expected. We can palpate the femoral artery from the body surface, and the femoral head exists near the point of palpating the femoral artery. Thus, we can predict the craniocaudal level of the orifice before the surgery. The orifice of the ureter was often found at the level of the upper portion of the femoral head in males and at the middle portion in females. In females, the uterus exists cranially to the urinary bladder, and we think this is the cause of the difference in orifice location between males and females. Dividing into the maximum bladder area, the orifice was higher in group 2 than in group 1 and 3 . The rate of males in group 2 (61.1\%) was higher than in group 1 (54.5\%) or 3 (45.5\%), so gender may be the underlying cause of the difference.

The mean transverse distance from the medial line of the body to the orifice of the ureterovesical junction was about $19 \mathrm{~mm}$, and was not significantly different between the right and left side. We suspected that the orifice was almost symmetric with regard to spine position. The distance was longer in females than in males. The ureter near the ureterovesical junction in the female is crossed anteriorly by the uterine artery just as this vessel turns medially to enter the base of the broad ligament [3]. This might be the cause of the difference in the distance. The distance was longer as the maximum area of the urinary bladder become larger. The bladder wall becomes distended with a larger bladder volume, and it may cause the difference.

On the basis of the results of the direction of the ureter from the ureterovesical junction, the ureter enters the bladder wall from the posterior, lateral, and cranial portion on the unenhanced CT in almost all patients. These 
findings match those of the prior report about the anatomy of the ureter [2]. Although the values of the $\varphi 1$ angle were between $60^{\circ}$ and $80^{\circ}$, and the $\varphi 2$ angle was between $40^{\circ}$ and $60^{\circ}$ in half and more of all patients, they had a wide range. In addition, in a minority of the patients, the ureter proceeded anterior from the ureterovesical junction. Thus, we suppose that evaluation of each individual case is important. The cause of difference of angles $\varphi 1$ and $\varphi 2$ between on the right and on the left side, and among 3 groups of the maximum bladder area is not clear. Compression from adjacent structures (for example, gastrointestinal tracts) may be the cause. The difference of angle $\varphi 1$ between females and males would be due to the presence of the uterine artery in females.

The mean length of the ureter from the point of turning medially to the ureterovesical junction was similar between the right and left sides in all patients. However, the values of the $\varphi 1$ and $\varphi 2$ angles had a wide range, so the point of turning medially would vary in each patient. The length was greater in females than in males. This difference might be related to difference of the transverse distance from the medial line of the body to the ureterovesical junction. Moreover, it may also be due to the uterine artery crossing the ureter. Dividing into the maximum bladder area, the mean length of the ureter was longer in group 2 than in group 1 and 3 . It might be due to the ratio of males and females.

This study has limitations. First, the ureters were not confirmed by surgery or autopsy. The invasive treatments are not undergo if we does not suspect urinary carcinomas, moreover urologists undergo preserving treatment for bladder cancer in our institution. At least, we assumed that the tubular or linear structure arising from the ureteropelvic junction, running through the intervening adipose tissue, and finally entering the bladder wall is the ureter on all preoperative CT, so the results of our study is feasible. Second, we investigated patients with hematuria or urothelial carcinomas, not those with rectal or cervical cancer. The results of this study may differ slightly from the situation in patients with rectal or cervical cancer. However, we excluded the patients with hydronephrosis, thus we suppose that populations in our study are similar to patients without diseases in the urinary systems, thus the results in our study would be applied to several patients with rectal or cervical cancer. Third, we did not consider that there are several tilts or rotation human at human pelvis due to degenerative disease or operation. The results may not be feasible for accurate anatomical data. However, the spine position on CT table is near the position of laparoscopic surgery, so the results of our study would be clinically useful. Forth, the volume of urinary bladder would be small due to urethral catheterization, thus the volume of the urinary bladder in this study may be different with that in the surgery. However, the maximum area was less than 1500 $\mathrm{mm}^{2}$ in group 1 , and the results in group 1 may be near to that of the urinary bladder on surgery. Finally, the number of patients in our study was small. The larger population may be needed for analyses.

\section{Conclusion}

We can evaluate the anatomy of the ureter near the ureterovesical junction on preoperative CT. According to the results, the ureterovesical junction exists at the level of upper or middle of the left femoral head. The direction of the ureter from the ureterovesical junction is usually posterior, lateral, and cranial, but has a wide range.

\section{Acknowledgements}

We thank to T. Sato MD for advising us about the anatomy of the pelvic plexus and its branch. CT was scanned by K. Ashida RT and S. Yoshikawa RT.

\section{References}

[1] Motola, J.A., Shahon, M.D. and Smith, A.D. (1988) Anatomy of the Ureter. The Urologic Clinics of North America, 15, 295-299.

[2] Frober, R. (2007) Surgical Anatomy of the Ureter. BJU International, 100, 949-965. http://dx.doi.org/10.1111/j.1464-410X.2007.07207.x

[3] Kaye, K.W. and Goldberg, M.E. (1982) Applied Anatomy of the Kidney and Ureter. The Urologic Clinics of North America, 9, 3-13.

[4] Sato, T. (1989) Kouza/Hinyoukika shujutu ni hituyou na kyokusho kaibou. 8III. Nyoukann (1). Rinnshou hinyoukika, 43, 111-119.

[5] Sato, T. (1989) Kouza/Hinyoukika shujutu ni hituyou na kyokusho kaibou. 9IV. Nyoukann (2). Rinnshou hinyoukika, 43, 241-249. 
[6] Jinzaki, M., Matsumoto, K., Kikuchi, E., Sato, K., Horiguchi, Y., Nishiwaki, Y. and Sliverman, S.G. (2011) Comparison of CT Urography and Excretory Urography in the Detection and Localization of Urothelial Carcinoma of the Upper Urinary Tract. American Journal of Roentgenology, 196, 1102-1109. http://dx.doi.org/10.2214/AJR.10.5249

[7] Juri, H., Matsuki, M., Inada, Y., Tsuboyama, T., Kumano, S., Azuma, H. and Narumi, Y. (2013) Low-Dose Computed Tomographic Urography Using Adaptive Iterative Dose Reduction 3-Dimensional: Comparison with Routine-Dose Computed Tomography with Filtered Back Projection. Journal of Computer Assisted Tomography, 37, 426-431. http://dx.doi.org/10.1097/RCT.0b013e3182830aa9

[8] Maas, C.O., Moriya, Y. and Steup, W.H. (1998) Radical and Nerve-Preserving Surgery for Rectal Cancer in the Netherlands: A Prospective Study on Morbidity and Functional Outcome. British Journal of Surgery, 85, 92-97. http://dx.doi.org/10.1046/j.1365-2168.1998.00530.x

[9] Pocard, M., Zinzindohoue, F., Haab, F., Caplin, S., Parac, R. and Tiret, E. (2002) A Prospective Study of Sexual and Urinary Function before and after Total Mesorectal Excision with Autonomic Nerve Preservation for Rectal Cancer. Surgery, 131, 368-372. http://dx.doi.org/10.1067/msy.2002.122371

[10] Fujii, S. (2008) Anatomic Identification of Nerve-Sparing Radical Hysterectomy: A Step-by-Step Procedure. Gynecologic Oncology, 111, S33-S41. http://dx.doi.org/10.1016/j.ygyno.2008.07.026

[11] Kuwabara, Y., Suzuki, M., Hashimoto, M., Furugen, Y., Yoshida, K. and Mitsuhashi, N. (2000) New Method to Prevent Bladder Dysfunction after Radical Hysterectomy for Uterine Cervical Cancer. Journal of Obstetrics and Gynaecology Research, 26, 1-8. http://dx.doi.org/10.1111/j.1447-0756.2000.tb01192.x 